\title{
Deformation Stress State of Elastic Bodies
}

\begin{abstract}
J. Skokánek
The theory of the deformation stress state is based on the actual corpuscular structure of matter characterized in terms of mechanics by the fact that an increase in the distance of two adjacent atoms is accompanied by the origin of an attractive force and a reduction in their distance by the origin of a repulsive force. These forces differ significantly from the classical internal forces, which are the forces of the mechanics of perfectly solid bodies. These express the equilibrium of forces with reference to the given area within the loaded body, and have no direct deformation effect. This paper defines the quantities of the deformation stress state - the deformation force and the deformation stress - the direct manifestation of which is a deformation. The author introduces the term of deformation stress state theory (DSS theory) to the field of the theory of elasticity dealing with the stress state of deformable bodies. The quantities and the equations of this theory also form the basis for the formulation of the theory of failure, which makes it possible to determine reliably the safety margin and the strength of a multiaxially loaded body from the stress state described by the static quantities (stress tensor) and uniaxial strength.
\end{abstract}

Keywords: deformation force, deformation stress, effective stress, effective strength, proportionality principle.

\section{Stress state of elastic bodies}

\subsection{Theory of elasticity uses forces and axioms of stereomechanics}

In the theory of elasticity the stress state of a loaded elastic body is described by the internal force and stress, i.e. quantities adopted from the mechanics of perfectly solid bodies. While the deformation depends on the physical and mechanical properties of the materials, and changes during any change of the load applied to the body, for a description of the stress state of elastic bodies we have only the quantities which do not depend directly either on the material properties or on the deformation magnitude of the body. From the mechanics of perfectly solid bodies the theory of elasticity adopts also both the fundamental axioms of statics, viz the axiom on the equilibrium of forces and the axiom on the composition and resolution of forces. According to the axiom on the equilibrium of forces, two forces are in equilibrium only if they act in opposite directions in a single line. According to the axiom on the composition and resolution of forces (axiom of the parallelogram of forces), the vector resolution or composition of forces does not change the state of equilibrium of the given system. A necessary prerequisite for the application of both of these axioms is the idealization of the continuous application of forces by a force concentrated in a beam. The concentration of the force in a single beam and its effect on a geometric point makes it possible to consider the force as a vector quantity and to use all vector operations.

According to Newton's third theorem (axiom on action and reaction), at every point of a contact surface between two bodies the vector of a specific load must equal the vector of the stress of the opposite direction. The effect of the forces on the contact of two bodies does not depend on their properties or on the changes in their volume and shape. Fig. 1. shows an interaction of the forces of two prismatic bodies of identical transverse dimensions on a contact surface from which friction has been excluded. In the direction of the first principal axis both bodies are affected by the load generating longitudinal principal stress $\sigma_{1}$ in them. The left body is exposed to a uniaxial load, while the right body is loaded additionally in the principal directions $x_{2}, x_{3}$ by a load generating transverse compressive stresses of equal magnitude $\sigma_{2}=\sigma_{3}=\sigma_{1}$. The equilibrium of the forces on the contact surface will manifest itself by the equal principal stress $\sigma_{1}$ acting from both of its sides. The deformations of the two bodies, however, are entirely different. The left body will be more compressed longitudinally than the right body. In the transverse direction the left body will elongate, while the right body will shorten. When the stress attains the strength in uniaxial compression, the left body will fail by a crack parallel with $x_{1}$, while the right body will not fail even under the highest values of external stresses.

The different deformations of the two bodies are shown illustratively by the deformation of a small spherical element
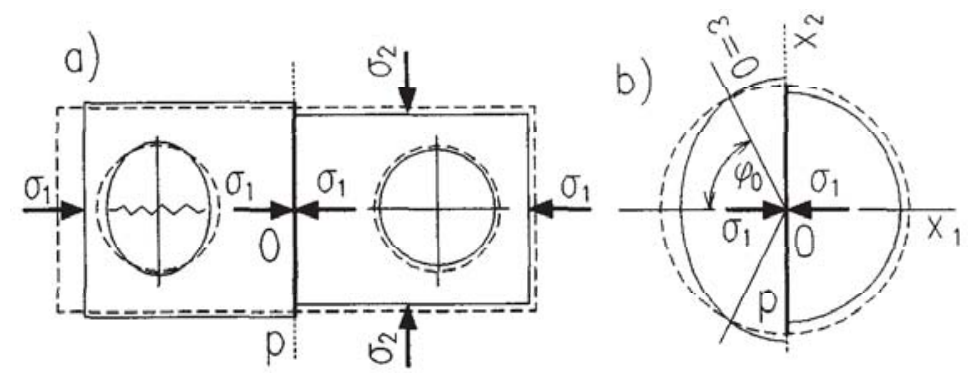

Fig. 1: Classical stress state and deformation state at the contact of two bodies subjected to different loads 
within the right and the left bodies (Fig. 1a). The initial shape of the elements is drawn by a dashed line, and the deformed shape by a solid line. While stress $\sigma_{1}$ has the same value in all cross sections of the two bodies perpendicular to $x_{1}$, the spherical element within the left body is deformed entirely differently from the spherical element within the right body. The different deformations of the two bodies are illustrated in Fig. 1b, showing the deformation of the two halves of the initially spherical element with the centre on their contact surface.

From the axiom of the composition and resolution of forces it follows that the two vectors of opposite directions of the resulting stress can be resolved into any number of components without disturbing the state of equilibrium or changing the deformation of either body. The resolution of the vectors does not influence the deformation state or the stress state. Fig. 2 shows a case of resolution of the result-
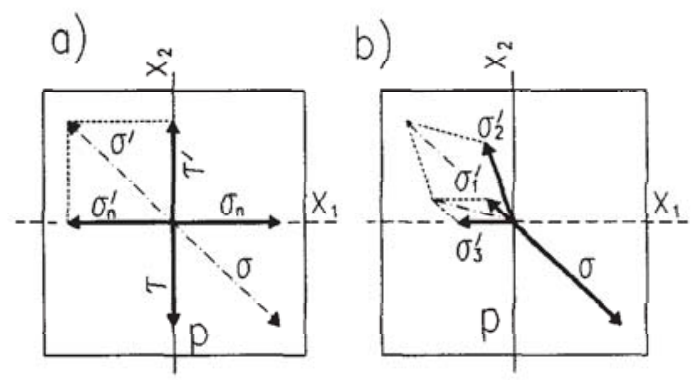

Fig. 2: Different cases of resolution of the resulting stress into its components

ing stress vector $\boldsymbol{\sigma}$ acting obliquely on a plane $p$ within the loaded body into various components. Fig. 2a shows the usual vector resolution into two components - normal $\sigma_{\mathrm{n}}$ and tangential $\tau$. Any stress vector resolution different on both sides of the surface does not change either the stress or the deformation. Fig. 2b shows one of the infinite number of cases of the possible resolution of the resulting stresses in the same stress state as in Fig. 2a. In the resulting stress direction of the right side, the stress component on the other side can have an entirely different magnitude. The resolution of every resulting stress vector acting on three mutually perpendicular planes into three components is useful for the definition of the three-dimensional stress state by means of the stress tensor; however, it is incorrect to assign to the individual stresses or even to their components any influence on the deformation (deformation effect).

\subsection{Erroneous opinion of the deformation effect of stress tensor components}

The relation between principle stresses and principal relative elongations is defined by the extended Hooke's law

$$
\begin{gathered}
\varepsilon_{1}=\frac{\sigma_{1}-v\left(\sigma_{2}+\sigma_{3}\right)}{E}, \\
\varepsilon_{2}=\frac{\sigma_{2}-v\left(\sigma_{3}+\sigma_{1}\right)}{E}, \\
\varepsilon_{3}=\frac{\sigma_{3}-v\left(\sigma_{1}+\sigma_{2}\right)}{E} .
\end{gathered}
$$

If two principal stresses equal zero, we speak about a uniaxial stress state. We obtain the relation between the single principal stress different from zero and the first principal elongation (basic Hooke's law) by the substitution $\boldsymbol{\sigma}_{2}=\boldsymbol{\sigma}_{3}=0$ in Eq. (1a)

$$
\varepsilon_{1}=\frac{\sigma_{1}}{E} .
$$

From the two equations (1b) and (1c) we obtain

$$
\varepsilon_{2}=\varepsilon_{3}=-\frac{v \sigma_{1}}{E}=-v \varepsilon_{1} .
$$

The general Hooke's law makes it obvious that every principal relative elongation depends on all principal stresses, and not only on the principal stress of identical direction. In the preceding paragraph we pointed out that none of the principal stresses will change due to any change in the other principal stresses. At the same time every change of one of the principal stresses will produce a change of the relative longitudinal deformation in every direction from the given point.

In the case of a multiaxial stress state, the relative elon-

Table 1

\begin{tabular}{|c|r|r|r|r|}
\hline Item & $\sigma_{1}$ & $\sigma_{2}$ & $\sigma_{3}$ & $E \varepsilon_{1}$ \\
\hline 1 & 0 & -100 & +100 & 0 \\
\hline 2 & 0 & 0 & +100 & -25 \\
\hline 3 & 0 & 0 & -100 & +25 \\
\hline 4 & 0 & -100 & -100 & +50 \\
\hline 5 & 0 & +100 & +100 & -50 \\
\hline 6 & -25 & -100 & -100 & +25 \\
\hline
\end{tabular}

gation in the direction of the given principal stress may attain different magnitudes depending on the other principal stresses. This is shown in Table 1 , which gives the relative elongation value (computed by means of the expanded Hooke's relation) in the zero stress direction for various values of the remaining two principal stresses. The selected modulus of elasticity is $E=1$, Poisson's coefficient $v=0.25$. Depending on the overall stress state, the relative elongations in the zero stress direction may have positive or negative values. Item 6 even shows a case when the body elongates in the compressive stress direction due to the effect of major transverse compressive stresses. In this case, during increasing stresses the body apparently paradoxically breaks in the compressive stress direction after achieving a certain limit of transverse compressive stresses.

These statements are confirmed by the tests made by Bridgman [1] with glass and steel bars installed in a high-pressure chamber in such a way that their ends protruded from the chamber (Fig. 3). The successively increasing pressure of the liquid increased both transverse principal stresses, while the longitudinal stress remained at zero level. When a certain limit of transverse compressive stress (pressure in the liquid) was attained, the bar broke in the zero stress direction. Bridgman was unable to explain the cause of this failure. He merely stated that the origin of the failure could not be explained by any known hypothesis. He considered the hypo- 


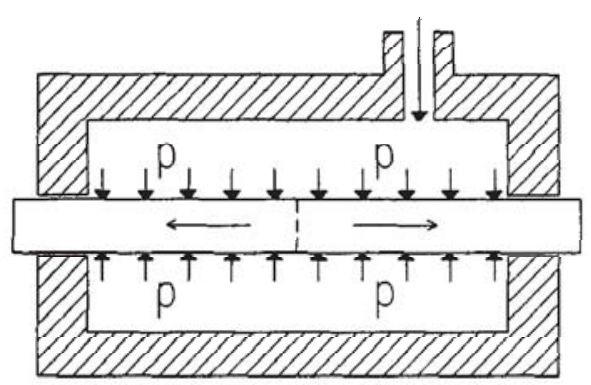

Fig. 3: Bridgman's test, in which a glass bar ruptures under the pressure of the liquid in the zero stress direction

thesis of maximum relative elongation to be the most likely, but his tests showed that the bars broke under the effect of markedly higher liquid pressure than those corresponding with this hypothesis.

To express the relation between the stress state and the further deformation stress state problems elaborated later on this paper, it is necessary to express the relative longitudinal change in any direction from the given point by a simple equation. The relative elongation in a general direction $\delta_{1}$, $\delta_{2}, \delta_{3}$ of any triaxially loaded body (Fig. 4) can be defined by means of given principal relative elongations $\varepsilon_{1}, \varepsilon_{2}, \varepsilon_{3}$ by the known equation

$$
\varepsilon=\varepsilon_{1} \cos ^{2} \delta_{1}+\varepsilon_{2} \cos ^{2} \delta_{2}+\varepsilon_{3} \cos ^{2} \delta_{3} .
$$

The equation for relative elongation in a general direction $\varphi$ of a uniaxially loaded body can be derived from this equation by putting $\delta_{1}=\varphi, \delta_{2}=\delta_{3}=\pi / 2-\varphi, \varepsilon_{2}=\varepsilon_{3}=-v \varepsilon_{1}$,

$$
\varepsilon_{\varphi}=\varepsilon_{1}\left[(1+v) \cos ^{2} \varphi-v\right]
$$

and by the substitution of $\varepsilon_{1}=\sigma_{1} / E$

$$
\varepsilon_{\varphi}=\frac{\sigma_{1}\left[(1+v) \cos ^{2} \varphi-v\right]}{E} .
$$

In a triaxially loaded body the relative elongation in a general direction expressed by angles $\delta_{1}, \delta_{2}, \delta_{3}$ can be determined by the superposition of the relative elongations produced by all uniaxial principal stresses

$$
\begin{aligned}
\varepsilon= & \frac{\sigma_{1}\left[(1+v) \cos ^{2} \delta_{1}-v\right]+\sigma_{2}\left[(1+v) \cos ^{2} \delta_{2}-v\right]}{E} \\
& +\frac{\sigma_{3}\left[(1+v) \cos ^{2} \delta_{3}-v\right]}{E} .
\end{aligned}
$$

It is more advantageous to express the relative elongation $\varepsilon$ in spherical coordinates $\varphi, \psi$, for which it holds that (Fig. 4)

$$
\begin{aligned}
& \cos ^{2} \delta_{1}=\cos ^{2} \varphi, \\
& \cos ^{2} \delta_{2}=\sin ^{2} \varphi \cos ^{2} \psi, \\
& \cos ^{2} \delta_{3}=\sin ^{2} \varphi \sin ^{2} \psi .
\end{aligned}
$$

Substitution in Eq. (6) yields the equation of relative elongation of a triaxially loaded body in a general direction

$$
\begin{aligned}
\varepsilon_{\varphi \psi}= & \frac{\sigma_{1}\left[(1+v) \cos ^{2} \varphi-v\right]}{E} \\
& +\frac{\sigma_{2}\left[(1+v) \sin ^{2} \varphi \cos ^{2} \psi-v\right]}{E} \\
& +\frac{\sigma_{3}\left[(1+v) \sin ^{2} \varphi \sin ^{2} \psi-v\right]}{E} .
\end{aligned}
$$

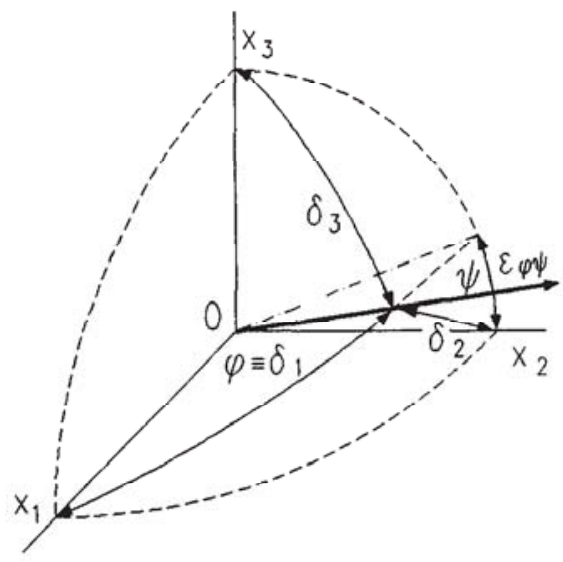

Fig. 4: Diagram of spheric coordinates

\subsection{Actual significance of shear stress}

In case of a general stress state, the vector of the resulting force is applied obliquely to a certain cross section area. In such a case the resulting internal force is usually resolved into the normal component and two tangential components. It follows from this that the stress expresses the equilibrium of forces with reference to the given cross section area, but has no direct influence on the longitudinal deformation in its direction. This applies, naturally, also to the orthogonal components into which we resolve the resulting stress vector according to the axiom of the parallelogram of forces. Neither the normal nor the tangential stress components have any deformation effect. Nevertheless, the deformation effect of the tangential stress components is generally considered as an indubitable fact, and exceeding the maximum shear stress is still considered a possible cause of failure. In the case of elastoplastic bodies, the origin of failure due to shear is even considered the most widely recognized failure hypothesis.

The themselves terms, such as shear stress, shearing force, shear area, shear failure and shear strength express the erroneous assumption that such a stress state produces a mutual displacement of two parts of the body. The shear effect of the shearing force is most frequently explained according to Fig. 5. For instance, "Strength of Materials" [4] states: "If external forces on the left-hand side of the cross section $p$ are composed into a resultant which has only tangential component $Q$ acting in the cross section plane with the point of application in the cross section centroid (the so-called shearing force) and the external forces on the right-hand side of infinitely near parallel cross section $p$ ' are composed according to the action and reaction theorem into a resultant $Q$ ' of opposite direction, of the same magnitude, with the point of application in the cross sections centroid, then both adjacent cross sections will be displaced mutually. When the ultimate 
shear strength has been attained, the cross section will be shorn off."
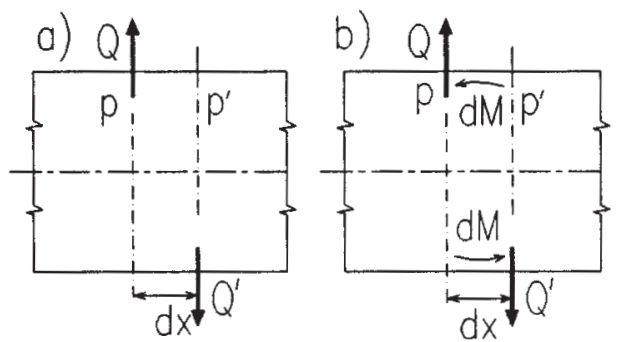

Fig. 5: Erroneous assumption of shear stress deformation effect according to [4]

This explanation of the shearing effect of the shearing force is based on the idea that both cross sections are infinitely near, so that it is possible to consider the opposite shearing forces as acting in a single plane, while assuming that their mutual distance enables mutual displacement. Such an assumption is evidently erroneous. If both shearing forces act at a differential distance dx according to Fig. 5, they produce a bending moment $\mathrm{d} M$ so that the equilibrium must be assured by a bending moment of equal magnitude, but of opposite direction. Thus $\mathrm{d} M=Q \cdot \mathrm{d} x$, hence

$$
Q=\frac{\mathrm{d} M}{\mathrm{~d} x},
$$

which is known as Schwedler's theorem, according to which the shearing force is the first derivative of the bending moment.

Also the statement that the cracks originating in the body at the ultimate strength of the material may have the direction of the principal shearing stresses, is erroneous. This is also shown by Fig. 6. Fig. 6a represents a body of cubic shape loaded by uniaxial pressure $\sigma_{2}$ in the direction of $x_{2}$. The
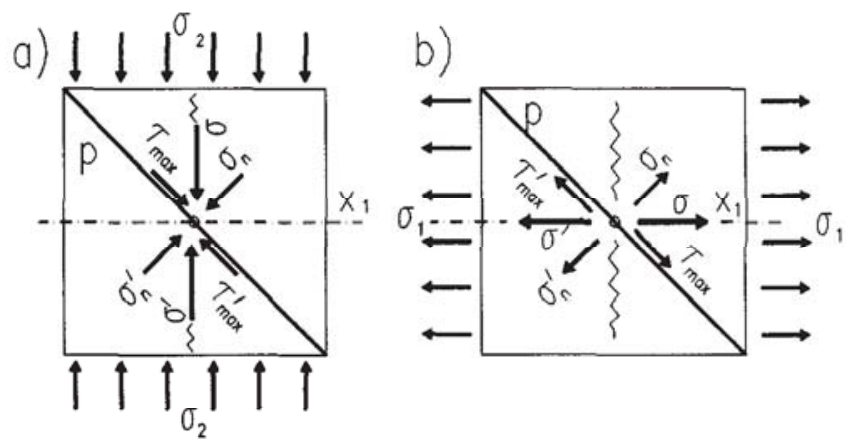

Fig. 6: The origin of a faillure crack does not depend on the magnitude of the shear stress, and its plane does not follow the direction of the maximum shear stress

maximum shear stress $\tau_{\max }$ acts on the plane $45^{\circ}$ diverting from $x_{2}$. The shear stress $\tau_{\text {max }}^{\prime}$ acts on the same plane in opposite direction as a reaction. The same maximum shear stress $\tau_{\text {max }}$ originates in the same plane of the same element during the uniaxial stress state produced by the tensile stress $\sigma_{1}=-\sigma_{2}$ acting in the direction of $x_{1}$ (Fig. 6b). Let us add that the tangential stress is not algebraically ambiguous (positive or negative), so that zero is considered its minimum value. Both cases involve a uniaxial load: in the former case in compression, and in the latter case in tension. In either case the failure crack does not originate in the shear stress direction, but in the direction perpendicular to $x_{1}$, which is the axis of maximum elongation in either case. The absolute values of both uniaxial strengths and the extreme shear values in both cases differ substantially. The uniaxial compression strength is several times as high as the uniaxial tensile strength, and the values of the ultimate shear stresses in both stress state cases are in the same ratio.

\section{Deformation stress state of elastic bodies}

\subsection{Influence of atomic structure on the deformation and stress state of solid bodies}

Classical stress is a plane force quantity of the mechanics of perfectly solid bodies expressing the equilibrium of forces applied to a unit surface from both sides. Stress exercises no direct influence on deformation. In the direction of a stress vector of a certain magnitude, the relative elongation may be of a different value and orientation, depending on the overall triaxial stress state (see Table 1). The principal stress at the contact of two bodies (Fig. 1) produces an entirely different three-dimensional deformation in each of them. An examination of the relative elongation in the given direction from the given point requires knowledge of the three stresses in the given point acting on three mutually perpendicular planes passing through the given point. An advantageous expression of the stress state is the stress tensor, the simplest (diagonal) form of which comprises three components - the principal stresses, by means of which it is possible to compute the principal relative elongations, according to Eq. (1), and the relative elongation in any direction from the given point in a

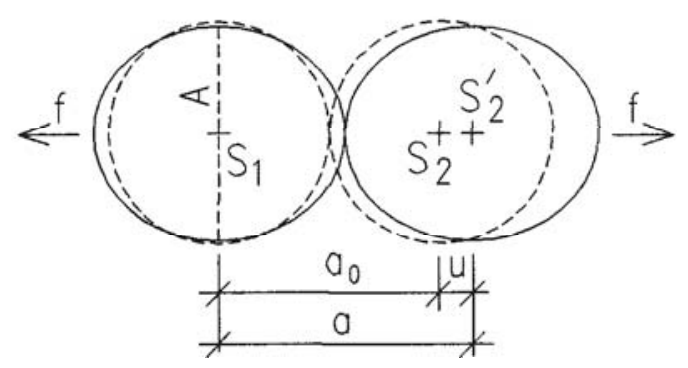

Fig. 7: The force acting between two atoms is accompanied by a change in their distance

triaxially loaded body according to Eq. (10). However, the solution of various problems of the theory of elasticity, particularly the examination of the multiaxial strength of bodies, lacks a force quantity which would be in a direct relation with the relative elongation. It is advisable to base the definition of such a quantity on the corpuscular structure of the bodies. The contemporary discrete theory of elasticity, which takes into account the atomic structure of bodies, is based on the assumption that between two atoms of a loaded body a repulsive force acts in the direction of their decreasing distance, 
and an attractive force acts in the direction of their growing distance (Fig. 7).

Cottrell [3] has come to the conclusion that the relation between the change of distance of two atoms $u$ and the force $f$ accompanying this change is linear. The equation expressing this relation can be written in the form

$$
f=\mathrm{c}_{\mathrm{k}} \cdot u,
$$

where $c_{k}$ is a proportionality coefficient.

Cottrell considers the proportion between force $f$ and longitudinal change $u$ in Eq. (12) to be the Hooke's law. Actually, however, there is a considerable difference between this equation and Hooke's law. According to Hooke's law, the relative longitudinal change in the direction of the principal axis of a uniaxially loaded body is proportionate to the internal force acting on the given plane surface (surface $\mathrm{A}$ in Fig. 7), perpendicular to the same axis. The atoms, however, affect their whole volumes by their forces; in this process their shapes and volumes change, as the picture illustrates.

Every atom has a major number of adjacent atoms. For instance, in a crystalline substance with a cubic face centered structure, every atom has 12 equally distant nearest neighbours (Fig. 8). The load changes the distances among all atoms, so that according to Eq. (12) every increase in the

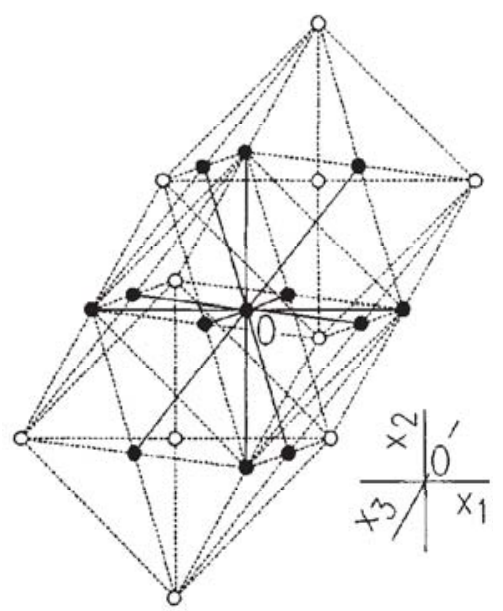

Fig. 8: Cubic face centered crystal structure

distance of the given atom from its neighbour is accompanied by an attractive force and by a reduction of their distance by a repulsive force. Cottrell [3] expressed the stress state of the loaded body by a mutual force effect of all its atoms, as follows: "The bulk elastic behaviour of large solid bodies is simply the aggregate effect of the individual deformations of the bonds in them, the applied force being transmitted from one loading point to another along the network of bonds running through the material". This statement reveals that the forces produced among atoms by the stress state are not plane internal forces, but the forces with deformation effect, i.e. deformation forces.

Fig. 9 shows the force effect of ambient atoms on a given atom and the changes in the distances of the atom centres in a biaxially compressed body. The boundaries of the electronic clouds of atoms in an unloaded state are idealized by spherical surfaces deformed under a load like microscopic three-dimensional elements. The picture simplifies the actual

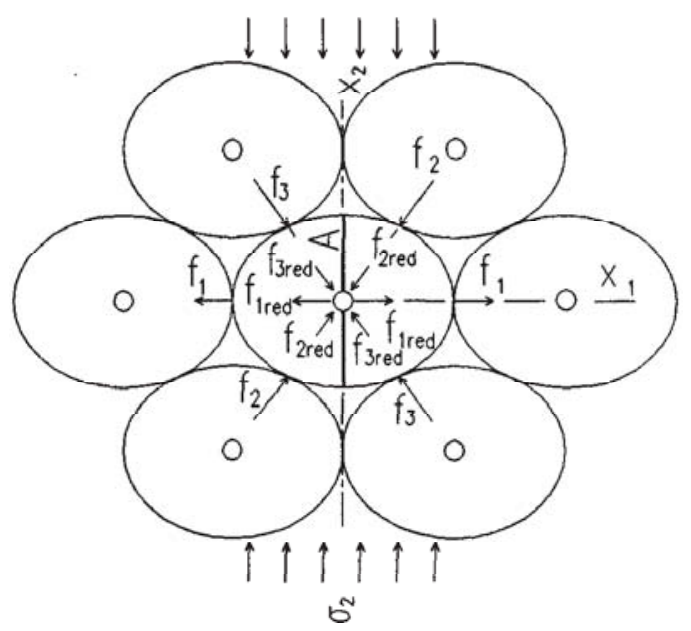

Fig. 9: Schematic diagram of the forces produced by biaxial compression between a given atom and its neighbours

spatial arrangement of the atoms in the crystal lattice by a two-dimensional arrangement, thus reducing also the actual number of adjacent atoms to the given atom. According to Eq. (12), the changes in distances of the centre of the given atom and all adjacent atoms are proportionate to the force originating among them in the process.

The biaxially loaded body will become narrower in the direction of the principal axes $x_{2}, x_{3}$ and will elongate in the direction of $x_{1}$. The spherical elements idealizing the boundary of the electronic clouds of atoms will narrow or elongate equally. In parallel with the deformation, the repulsive forces $f_{2}, f_{3}$ and the attractive force $f_{1}$ will originate between the given atom and its neighbours. When we examine the resulting internal force $\boldsymbol{F}_{1}$ acting on the cross section area $A$ of the given atom perpendicular to $x_{1}$, we must add the vector effect of all interatomic forces on this surface. Generally speaking, the forces $f$ affect the area $A$ obliquely only by reduced values for which it holds that

$$
f_{\text {red }}=f \cdot \cos \varphi \text {. }
$$

The resulting internal force $\boldsymbol{F}_{1}$ is then the vector sum of all partial internal forces acting on the given area from one side

$\boldsymbol{F}_{1}=\sum_{i=1}^{n} f_{\mathrm{red}, i} \cdot \cos \varphi_{i}=\sum_{i=1}^{n} f_{i} \cdot \cos ^{2} \varphi_{i}$ for $i=1$ to $n$.

The schematic diagram also reveals why in the case of biaxial compression the internal force in direction $x_{1}$ equals zero, although the body elongates in the same direction. This is due to fact that the magnitude of the internal force $\boldsymbol{F}_{1}$ is participated in by both positive and negative internal forces. The sum of their normal components is in this case the zero internal force.

The idealization of the forces acting on the given atom, accompanied by the change in its distance from the adjacent atoms, gives only an approximate picture of the force effects among the atoms. This is due to the fact that the forces $f_{i}$ are discrete quantities, cumulating the deformation force effect on the given three-dimensional element (atom) from a certain spatial angle into one vector, while the relative elongation changes continuously within the same spatial angle. 
According to this model, the number of forces $f$ is limited by the number of the nearest neighbours of the given atom. The forces acting linearly between the atoms are based on the idealization of a three-dimensional material element (an atom in this particular case) by a material point. Actually, the force interaction of the given atom with every one of its neighbours is realized by the interaction of all their particles, particularly protons with a positive charge and moving electrons with a negative charge. The charges with the same sign repel one another, while the charges with different signs attract one another.

The load changes the shape and volume of the electron cloud and, consequently, the distance of the atoms, giving rise to an electromagnetic field which manifests itself by the continued force effect on the given atom simultaneously with a continuous change in its shape and dimensions. We speak about the deformation force effect.

\subsection{Dependence of classical stress on the deformation stress state}

At a small distance from a given point it is possible to consider the relative elongation in a given direction as constant. Therefore, when examining the dependence between a deformation and stress state in the environs of a given atom, we can replace the force effect on the atom with the stress state of a macroscopic spherical element of very small radius with its centre coinciding with the centre of the atom. The strain state of a spherical element of unit radius gives the elongation of the radius in all directions the magnitude of the relative elongation. Fig. 10a shows the deformation of a spherical element of unit radius loaded by uniaxial tension, while Fig. 10b is a schematic representation of the deformation force effect on the same element. Within the scope of the differential solid angle $\mathrm{d} \Omega$ (Fig. 10c), the deformation force $\mathrm{d} D$ acts on the area of the element surface $\mathrm{d} A_{\mathrm{sf}}=r^{2} \mathrm{~d} \Omega$ (for the radius $r=1$ it is $\mathrm{d} D=\mathrm{d} D_{1}$ and $\mathrm{d} A_{\mathrm{sf}}=\mathrm{d} \Omega$ ). For the quota of deformation force $\mathrm{d} D_{1}$ and the spherical area $\mathrm{d} A_{\mathrm{sf}}=\mathrm{d} \Omega$ affected by it we shall introduce the term deformation stress $(\vartheta)$

$$
\vartheta=\frac{\mathrm{d} D}{\mathrm{~d} A_{\mathrm{sf}}}=\frac{\mathrm{d} D_{1}}{\mathrm{~d} \Omega} \quad\left[\mathrm{N} / \mathrm{mm}^{2}\right] .
$$

Like the elementary deformation force, the deformation stress must also be positive in the direction of the positive elongation, negative in the direction of relative shortening, and equal to zero in the direction of zero elongation. When taking into account the corpuscular structure of bodies, the shear stress has no real meaning. The definition of deformation stress will reduce the relation (12) to the proportionality between stress and relative longitudinal change

$$
\vartheta=c \cdot \varepsilon,
$$

where $c$ is the coefficient of proportionality between deformation stress and relative elongation.

If we substitute $\varepsilon_{\varphi}$ from Eq. (5) in Eq. (16), we obtain the equation for deformation stress in a general direction of a uniaxially loaded body

$$
\vartheta_{\varphi}=c \sigma_{1} \frac{(1+v) \cos ^{2} \varphi-v}{E} .
$$

After the substitution of $\varepsilon_{\varphi}$ from Eq. (10) in Eq. (16), the deformation stress equation in general direction of a triaxially loaded body will acquire the form

$$
\begin{aligned}
\vartheta_{\varphi \psi}= & \frac{c}{E}\left\{\sigma_{1}\left[(1+v) \cos ^{2} \varphi-v\right]\right. \\
& +\sigma_{2}\left[(1+v) \sin ^{2} \varphi \cos ^{2} \psi-v\right] \\
& \left.+\sigma_{3}\left[(1+v) \sin ^{2} \varphi \sin ^{2} \psi-v\right]\right\} .
\end{aligned}
$$

The equation expresses the deformation force effect on the spherical element of a triaxially loaded body, for which we shall introduce the term deformation stress state (DSS). By putting $\vartheta_{\varphi \psi}=0$ and $\psi=0$, or $\pi / 2$, we obtain from this equation the formulas for computation of the angles of zero deformation stresses in the planes $x_{1} x_{2}$ and $x_{1} x_{3}$ for a triaxially loaded body

$$
\cos ^{2} \varphi_{0(12)}=\frac{\nu\left(\sigma_{1}+\sigma_{3}\right)-\sigma_{2}}{(1+v)\left(\sigma_{1}-\sigma_{2}\right)}
$$
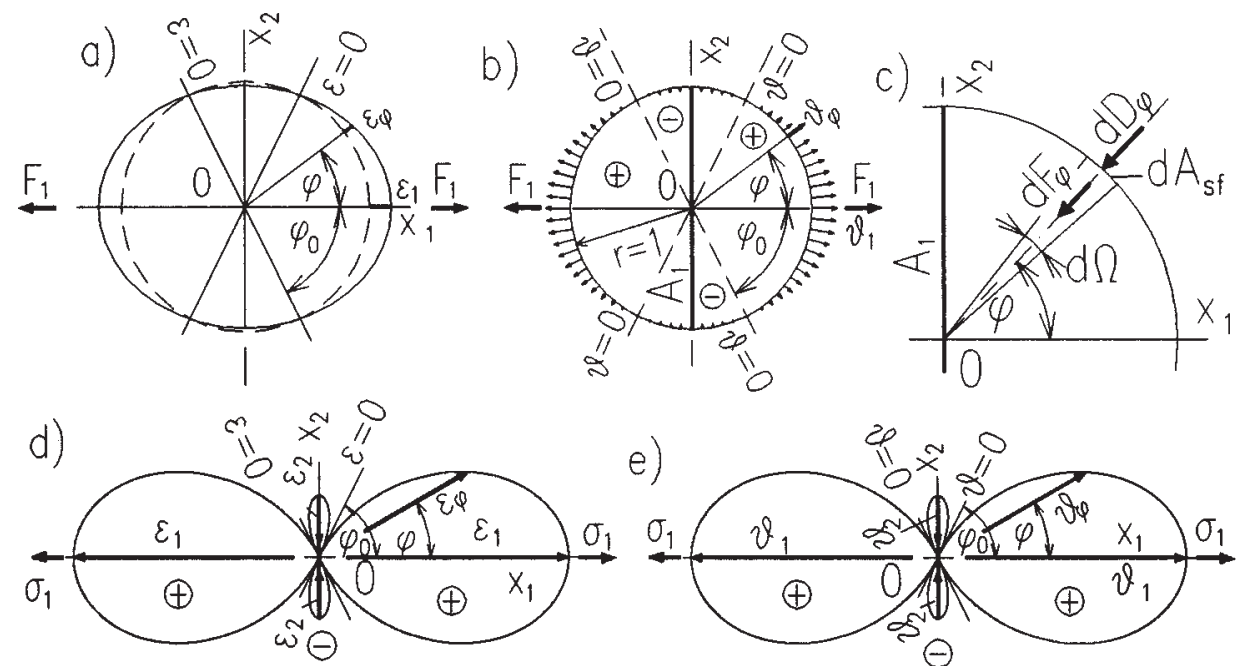

Fig. 10: Strain and deformation stress state in a uniaxially loaded body 


$$
\cos ^{2} \varphi_{0(13)}=\frac{v\left(\sigma_{1}+\sigma_{2}\right)-\sigma_{3}}{(1+v)\left(\sigma_{1}-\sigma_{3}\right)} .
$$

In a uniaxially loaded body, the directional cosine of zero elongation is obtained from Eq. (5), where we shall substitute $\varepsilon_{\varphi}=0$

$$
\cos \varphi_{0}=\left(\frac{v}{1+v}\right)^{\frac{1}{2}}
$$

A more adequate graphic representation of the deformation state and the deformation stress state than that given by Figs. 10a and 10b is provided by the deformation and the deformation stress state curves (Fig. 10d and 10e), which are a graphic representation of Eqs. (5) and (17). The curves are geometrically similar.

The definition of the continuous deformation force effect enables a refinement of the relation (14) between the internal force and the interatomic forces, based on the assumption of their linear effect. Instead of summing up the normal components of the individual reduced interatomic forces acting on the surface perpendicular to the first principal axis, we shall integrate the normal components $\mathrm{d} N_{\varphi}$ of the reduced elementary deformation forces $\mathrm{d} F \varphi$ (Fig. 10c)

$\mathrm{d} N_{\varphi}=\mathrm{d} F_{\varphi} \cos \varphi=\vartheta_{\varphi} \cos ^{2} \varphi \mathrm{d} A_{\mathrm{sf}}=\vartheta_{\varphi} \cos ^{2} \varphi \mathrm{d} \Omega$.

The normal force $N_{1}$ identical with the resulting internal force $F_{1}$, affecting surface $A_{1}$ in this particular case, is

$F_{1}=\int_{2 \pi} \vartheta_{\varphi} \cos ^{2} \varphi \mathrm{d} A_{\mathrm{sf}}=\int_{2 \pi} \vartheta_{\varphi} \cos ^{2} \varphi \mathrm{d} \Omega$.

In the spherical element of unit radius $A_{1}=\pi$, so that the first principal stress is

$$
\sigma_{1}=\frac{1}{\pi} \int_{2 \pi} \vartheta_{\varphi} \cos ^{2} \varphi \mathrm{d} \Omega .
$$

If we substitute in Eq. (23) $\vartheta_{\varphi}$ from Eq. (17) for the uniaxial deformation stress state and put $v=0.25$ which is in the theory of DSS, according to [7] is Poisson's coefficient of linearly elastic materials, the solution yields the proportionality coefficient $c=2 E$, the substitution of which in Eq. (16) yields

$$
\vartheta=3 E \varepsilon \text {. }
$$

This equation holds for any, i.e. also for a triaxially loaded linearly elastic body. This is the fundamental relation of the deformation stress state theory, which we call the principle of proportionality: in any loaded linearly elastic body the defor- mation stress is proportionate to the relative longitudinal change.

Let us return to the deformation force effect on the spherical element in a uniaxially loaded body (Fig. 10). In the space defined by conical surfaces of zero longitudinal deformation (Eq. 20), the axis of which coincides with the first principal axis, only positive longitudinal changes take place. This means that within the volume of the element defined by this surface, the element is affected only by positive deformation forces. The remaining part of the element volume is loaded by negative deformation forces. The cross section through the spherical element $\left(A_{1}=\pi\right)$ perpendicular to the first principal axis is affected on one side by internal force $F_{1}$, which must be in equilibrium with the resultant of the deformation force effect on the adjacent half of the element volume. Let us note that the half of the element above $A_{1}$ is affected by both positive and negative deformation forces. The internal force $F_{1}$ (Eq. 22), consequently, cumulates not only the positive effect of the forces affecting the elongation of the element, but also the negative deformation force effect producing its compression. However, the elongation of the element and its rupture in the ultimate stress state is influenced only by the positive part of this force effect; the negative force effect produces transverse compression of the element. The principal Hooke's law (Eq. 2a), consequently, does not represent the relation between the positive force effect on $A_{1}$ and the relative elongation $\varepsilon_{1}$, but the relation characterizing the summary effect of the positive and negative force effect on $A_{1}$.

The deformation and the deformation force effect on the spherical element are independent of the orientation of the cross section surface. For a uniaxially loaded body this is clearly illustrated by Fig. 11. The deformation stress state is not changed by any change of location of the cross section surface. However, every change in the position of the surface does change the magnitude and, usually, also the direction of the internal force. Fig. 11a shows the equilibrium of the internal forces with reference to the surface perpendicular to the first principal axis (see also Fig. 10b). If the surface is not perpendicular to any of the principal axes, the resulting stress affects it obliquely (Fig. 11b). In this case we usually express the equilibrium of the forces with reference to the cross section surface by means of the normal and tangential components $(N, T)$ of the resulting force $F$. If we select the cross section surface parallel with the first principal axis, the inter-
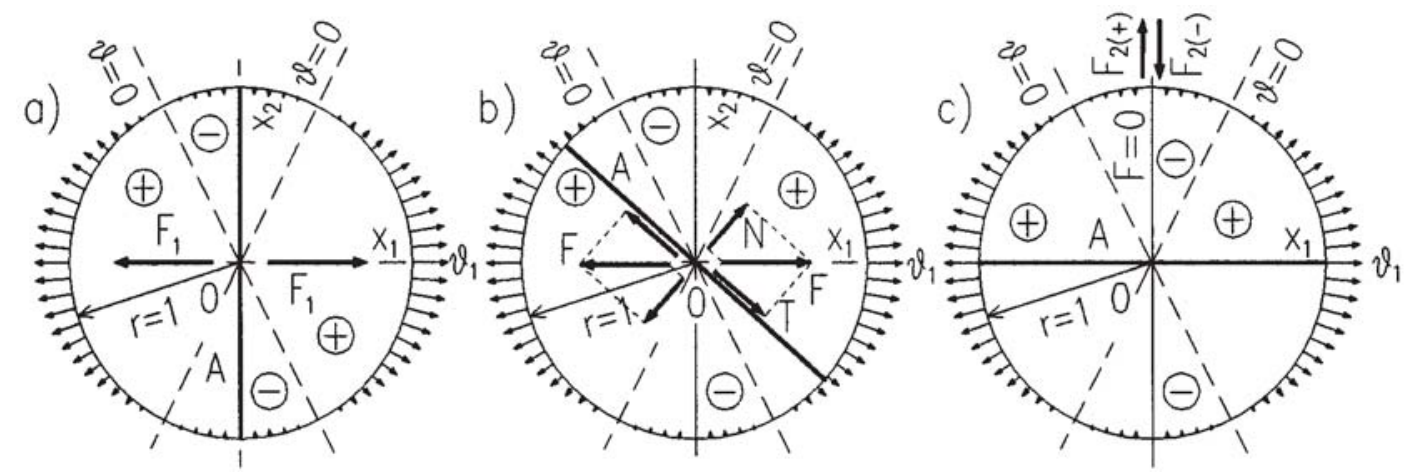

Fig. 11: The internal force changes with a change in the position of the cross section surface, while the deformation stress does not change 
nal force $F_{2}$ referred to it, is equal to zero (Fig. 11c). This means that the positive part of the internal force $F_{2(+)}$ generated by the positive deformation force effect on the half of the element above the cross section surface is in equilibrium with the negative part of the internal force $F_{2(-)}$ generated by the negative deformation force effect

$$
F_{2(+)}+F_{2(-)}=0 \text {. }
$$

Although the relative longitudinal change in the direction of $x_{2}$ has a value different from zero $\left(\varepsilon_{2}=-v \varepsilon_{1}\right)$, the stress in the same direction is equal to zero. By analogy it is possible to explain also other stress states that are otherwise difficult to explain, in which the vector of relative elongation in one direction may have even an opposite orientation than the resulting stress vector (see Table 1).

\section{Fields of practical application of deformation stress state theory}

Deformation stress state theory covers an extensive field of the theory of elasticity. The limited scope of this paper can provide only basic information on the deformation stress state and its relation to the stress state expressed by classical stress state quantities - stress and its components. It provides a new look at the stress state as a phenomenon accompanying deformation. The application of new deformation stress state quantities will enable the solution of a number of problems in the theory of elasticity for the solution of which the application of existing force quantities of stereomechanics is inadequate. These include, in particular:

- analysis of triaxial strength and determination of the safety margin of elastic bodies,

- analysis of the multiaxial strength of technical materials,

- clarification of the origin of the deformation and failure of triaxially loaded elastoplastic bodies,

- analysis of the strength and yield limit of three-dimensionally loaded elastoplastic bodies.

Of extraordinary significance for practical application is the formulation of the theory of failure of elastic and elastoplastic materials. The principal problem in existing hypotheses of the origin of failure is erroneous selection of the criterial quantity. Although it is known that failure originates due to exceeding a certain limit state of a three-dimensional stress state, which can be defined only by a complete stress tensor, i.e. by all its components, most hypotheses use only its individual components as the criterion of failure.
The theory of failure derived from deformation stress state theory is based on the following assumptions:

- Failure can be due only to rupture of the body; the assumption that the origin of failure is due to shearing or crushing, i.e. shear or compressive stress, is erroneous.

- The origin of failure is influenced only by the positive part of the deformation stress state accompanied by positive three-dimensional deformation. The negative part of the deformation stress state affecting compression has no direct influence on the origin of failure.

- The failure crack is always perpendicular to the vector of the maximum principal stress.

For the positive part of the maximum principal stress $\sigma_{1(+)}$ we have introduced the term effective stress $\Phi$, and for the positive part of the maximum internal force $F_{1(+)}$ the term effective force $F_{\Phi}$. The effective stress equation is obtained from Eq. (23), in which the solid angle of the deformation stresses with reference to the cross section surface $(A=\pi)$ is limited to the solid angle of the positive deformation force effect $\Omega_{(+)}$(Fig. 12).

$$
\Phi=\frac{1}{\pi} \int_{\Omega_{(+)}} \vartheta_{\varphi} \cos ^{2} \varphi \mathrm{d} \Omega .
$$

The effective stress indicates the magnitude of the stress state. Failure originates in any three-dimensional stress state by exceeding the same maximum effective stress value, effective strength $f_{\varphi}$, i.e. the ultimate positive part of the resulting stress with reference to the surface perpendicular to the principal axis of maximum elongation.

The criterion of failure based on deformation stress state theory is expressed as follows: failure occurs in that point of the body in which the maximum effective stress $\Phi_{\max }$ attains effective trength $f_{\Phi}$

This criterion yields the limit condition

$$
\Phi_{\max }<f_{\Phi} .
$$

The effective strength is obtained from any classical strength value (preferably strength in uniaxial compression or tension) obtained by tests and expressed by classical stress. Fig. 12 shows the deformation force effect under various loads applied to a spherical element. Fig. 12a shows schematically the deformation stress state of a biaxially compressed element. The positive part of the deformation stress state is limited by the conical surface of zero deformation stress $\vartheta=0$, which is also the surface of zero relative elongation $\varepsilon=0$. The

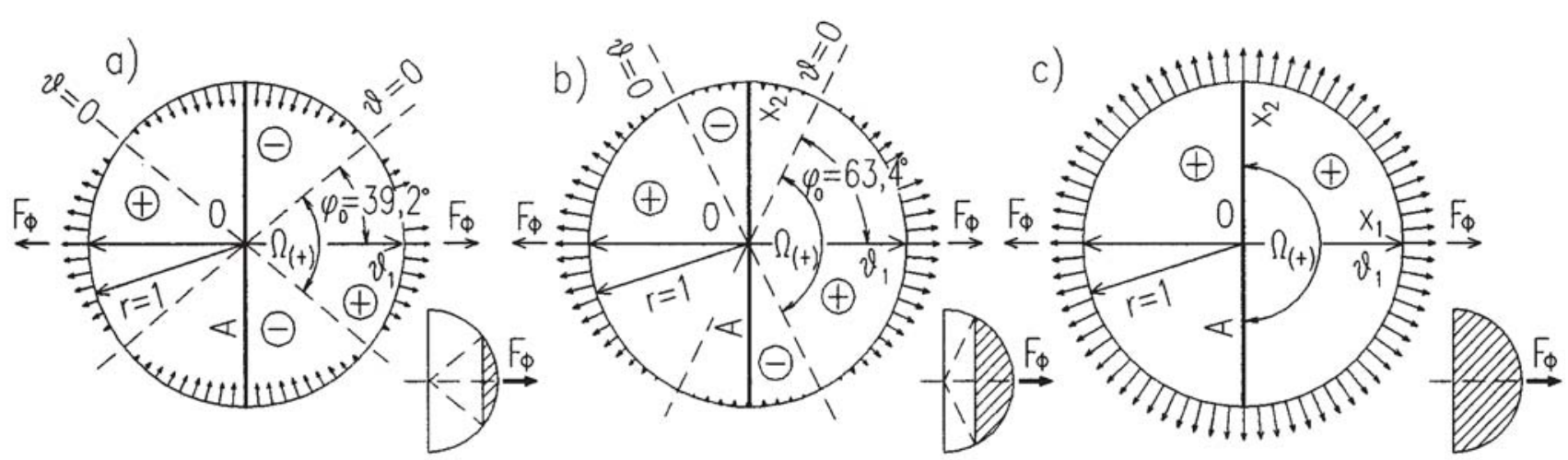

Fig. 12: Schematic diagram of the deformation force effect on the spherical element in the classical stress state 
picture also explains why in the case of biaxial compression the body ruptures in the direction of zero stress. The rupture is due to the fact that the value of the positive part of the internal force is equal to the absolute value of its negative part (Eq. 25). Although the internal force is equal to zero, the body ruptures as soon as the effective stress attains the value of the effective strength.

Figs. $12 b$ and 12c show schematically the deformation stress state of the element subjected to uniaxial tension and of the element subjected to uniform all-direction tension, respectively. The small diagrams attached to the pictures show that for the maximum deformation of the same magnitude $\vartheta_{1}$ (and, consequently, also maximum relative elongation $\varepsilon_{1}$ ), the effective force $F_{\Phi}$ is smallest under biaxial compression and greatest under uniform all-direction tension. This explains why, when the effective strength $f_{\Phi}$ has been attained, the relative elongation is highest under biaxial compression and lowest under all-directional tension.

\section{References}

[1] Bridgman, P. W.: The Physics of High Pressure, chapter 4. London, G. Bell and Sons Ltd., 1954.
[2] Bridgman, P. W.: "Collected Experimental Papers", Papers 1-14, Cambridge, Massachussets, Harvard University Press, 1964.

[3] Cottrell, A. H.: The Mechanical Properties of Matter. New York, London, Sydney, J. Wiley and Sons, Inc. 1964.

[4] Novák, O. et al.: "Strength of Materials in Building." Technical Guidebook Vol. 3 (in Czech), Prague, SNTL Publishers, 1963.

[5] Servít, R.: "Statics of Building Structures" Technical Guidebook (in Czech), Prague, SNTL Publishers, 1973.

[6] Servít, R.: Strength of Materials in Construction, Vol. II Theory of Failure (in Czech), Prague, SNTL Publishers, 1966.

[7] Skokánek, J.: "Deformation Stress State Theory." (in Czech), Prague 2004, unpublished.

Ing. Jiří Skokánek, CSc.

autorizovaný inženýr pro statiku a dynamiku staveb phone: +420241727378

Korandova 37

14700 Praha 4, Czech Republic 